\title{
The impact of the recipient and donor interferon lambda-3 polymorphism on the course of HCV infection following liver transplantation
}

\author{
Renata Wieczorek-Godlewska' ${ }^{1}$ Rafał Płoski ${ }^{2}$, Agnieszka Perkowska-Ptasińska' ${ }^{1}$, Olga Tronina' ${ }^{1}$ Anna Sadowska' \\ Marek Pacholczyk3, Wojciech Lisik³ ${ }^{3}$ Magdalena Durlik'
}

'Department of Transplantation Medicine and Nephrology, Medical University of Warsaw, Poland

${ }^{2}$ Medical Genetics Unit, Medical University of Warsaw, Poland

${ }^{3}$ Department of General and Transplant Surgery, Medical University of Warsaw, Poland

\begin{abstract}
Aim of the study was to assess the impact of the recipient and donor interferon lambda-3 (IFNL3) single-nucleotide polymorphisms (SNPs) rs12979860 and rs8099917 on the course of hepatitis C virus (HCV) reinfection following liver transplantation.

Material and methods: The study involved 141 subjects after liver transplantation for HCV-induced cirrhosis, performed between 2000 and 2015. It assessed the impact of both SNPs on the outcomes of interferon/ribavirin (IFN/RBV) treatment following transplantation, HCV viral load, laboratory test results, histological lesions in the liver graft, the risk of acute rejection, and the development of hepatocellular carcinoma (HCC) in patient's own liver.

Results: In the case of rs 12979860 , SVR was achieved in $58.8 \%$ of recipients with the CC genotype, and only $12 \%$ of recipients with the $\Pi$ genotype $(p=0.016)$. Recipients with the rs $12979860 \mathrm{CC}$ variant had lower viral load and lower alanine transaminase (ALT) activity than recipients with a non-CC variant. Opposite effects were demonstrated in the analysis of the donors' genotype. Recipients with the unfavorable variants (rs12979860 TT and rs8099917 GG) had a lower risk of graft rejection and tended to have a higher risk of developing HCC in their own liver.

Conclusions: The IFNL3 rs12979860 polymorphism may be considered a predictor for IFN/RBV effectiveness following liver transplantation. The course of HCV reinfection following liver transplantation may be more aggressive if an unfavorable variant in the recipient coexists with a promising variant in the donor. Particularly careful monitoring for $\mathrm{HCC}$ in recipients with unfavorable IFNL3 variants is warranted.
\end{abstract}

Key words: interferon lambda-3 (IFNL3), hepatitis C virus (HCV), liver transplantation.

\section{Address for correspondence}

Renata Wieczorek-Godlewska, Department of Transplantation Medicine and Nephrology, Medical University of Warsaw, 59 Nowogrodzka St., 02-006 Warszawa, Poland, phone: +48 22502 12 32, fax: +48 2250221 26, e-mail: godlewska.r@wp.pl

\section{Introduction}

In present-day, cirrhosis of the liver induced by chronic infection with hepatitis $\mathrm{C}$ virus (HCV) is the most common indication for liver transplantation in adults living in the developed countries [1]. The prognosis for this patient population is, however, much worse as compared with other indications, which is related primarily to frequent $\mathrm{HCV}$ reinfection in the donor's liver, occurring in nearly $95 \%$ of patients with detectable $\mathrm{HCV}$ prior to transplantation [2, 3]. The course of HCV reinfection is more aggressive than infection in the patient's own liver, with more rapid progression of fibrosis and development of cirrhosis 
of the graft [4]. Also outcomes of treatment of HCV reinfection in the liver graft using standard pegylated interferon (IFN) and ribavirin are clearly worse than in the case of the patient's own liver: permanent eradication of the virus (sustained virological response - SVR) is achieved only in $24-40 \%$ of the treated patients [5-8].

In genome-wide association studies (GWASs) in HCV-infected individuals, single nucleotide polymorphisms (SNPs) were identified and located in the region of IFNL3 (IL28B), which encodes antiviral cytokine interferon lambda 3 (IFNL3, IL28B, interleukin 28B), and is associated with the effectiveness of the interferon/ ribavirin treatment. The highest prognostic value was seen in the case of two loci: rs12979860 C > T and rs8099917 G > T, and the genotypes correlating with cure were: CC for rs12979860 and TT for rs8099917 [9-12]. Since the source of IFNL3 is composed of both immune cells (especially plasmacytoid dendritic cells [pDCs] and macrophages) and hepatocytes, chimerism occurs after liver transplantation. The patient has two sources of IFNL3: their own immune cells and the donor's hepatocytes, which may affect the immune response to HCV infection following transplantation [13].

The aim of this study is to assess the impact of the recipient and donor IFNL3 single-nucleotide polymorphisms (SNPs) rs12979860 and rs8099917 on the course of $\mathrm{HCV}$ reinfection following liver transplantation.

\section{Material and methods}

The study involved 184 subjects after liver transplantation for HCV-induced cirrhosis performed between November 2000 and February 2015 at the Department of General and Transplant Surgery, Medical University of Warsaw.

Patients who died within 90 days of transplantation $(n=19)$, patients with undetectable blood HCV RNA prior to transplantation $(n=9)$, and patients infected with HCVG3 $(n=15)$ were excluded from the final analysis. The proper group comprised 141 individuals with HCV reinfection in the liver graft, confirmed by detectable blood HCV RNA following transplantation, infected with HCV genotype $1(n=137)$ or $4(n=4)$. Mean follow-up time was $6.6 \pm 3.9$ years, ranging between 15.2 years and 1 year.

The study was approved by the Ethics Committee of the Medical University of Warsaw.

\section{IFNL3 genotyping}

The recipients' genetic material for IFNL3 polymorphism testing was obtained from fragments of the patients' own liver removed during transplantation and stored as paraffin-embedded blocks. The donors' genetic material was obtained through time-zero biopsies preceding reperfusion and was stored as paraffin-embedded blocks.

DNA was isolated using the Maxwell 16 (Promega GmbH, Germany) instrument for nucleic acid and protein isolation. The Maxwell ${ }^{\circ} 16$ FFPE Tissue LEV DNA Purification Kit (Promega GmbH, Germany) was used. Nucleotide sequences within rs12979860 were determined using allele-specific probes and the Custom TaqMan ${ }^{\circ}$ SNP Genotyping Assay (Applied Biosystems, A Thermo Fisher Scientific Brand). Possible single-nucleotide polymorphisms (SNPs) C $>$ T within rs 12979860 included CC, CT, and TT. IFNL3 rs8099917 G > T genotyping was performed using the TaqMan ${ }^{\circledR}$ Pre-Designed SNP Genotyping Assay (Applied Biosystems, A Thermo Fisher Scientific Brand). Possible SNP variants included TT, GT, and GG.

IFNL3 rs12979860 genotyping was performed for $88 \%$ of the recipients and $96 \%$ of the donors as well as for 120 recipient-donor pairs. In the case of the rs 8099917 polymorphism, successful genotyping was performed for $84 \%$ of the recipients and $96 \%$ of the donors as well as 115 recipient-donor pairs.

\section{Histological examinations}

The total of 368 biopsies performed in the study group between November 2000 and December 2015 were included in the analysis. The biopsies were performed 12, 24, and 36 months after transplantation, both for protocol-related reasons and because of clinical indications. Necroinflammatory lesions related to HCV reinfection (grading) and fibrosis severity (staging) were assessed using the Ishak scale [14]. Every biopsy was also assessed for acute liver graft rejection using the Banff 1997 classification [15]. The removed fragments of the patients' own livers were also assessed for hepatocellular carcinoma (HCC).

\section{Antiviral treatment following liver transplantation}

In 76 of 141 (54\%) patients diagnosed with HCV reinfection of the liver graft, double antiviral therapy with pegylated interferon alpha (PegIFN-a2a or PegIFN- $\alpha 2 b$ ) and ribavirin (RBV) was used. Indications for treatment initiation as well as doses and treatment duration met the then required standards of the American Association for the Study of Liver Diseases (AASLD) and European Association for the Study of the Liver (EASL), and were consistent with the guidelines set out by the Polish Group of Experts on HCV (PGE HCV). 
Treatment was considered successful if no viral load was detectable 24 weeks after treatment completion, i.e. when sustained virologic response (SVR 24) was achieved.

\section{Statistical methods}

Continuous variables were presented descriptively as the mean and standard deviation for normal distribution or median and quartiles [1Q-3Q], if the distribution was inconsistent with normal distribution. For normality testing, the Shapiro-Wilk test was used. In the case of categorical variables, the non-parametric Mann-Whitney $U$ test was used. To verify hypotheses on the rates of categorical variables in two subgroups, the $\chi^{2}$ test was used. To compare the means in more than two subgroups, non-parametric analysis of variance using the Kruskal-Wallis test was carried out. All hypotheses were tested using the significance level of 0.05 . The statistical analysis was carried out using $R$ under the GNU GPL license (version 3.2.0).

\section{Results}

The distribution of IFNL3 genotypes in the donors and recipients was significantly different for both SNPs of interest. Favorable (from the point of view of cure) $\mathrm{HCV}$ genotypes were more common in the donors than in the recipients: for rs 12979860 , the CC variant was found in $23.4 \%$ of the recipients and $38.5 \%$ of the donors; $p=0.013$; for rs 8099917 , the TT variant was found in $49.6 \%$ of the recipients and $64.6 \%$ of the donors; $p=0.023$.

An analysis concerning individual genotypes (CC, CT, and TT for rs12979860 and TT, GT, and GG for rs8099917) did not reveal differences in the recipients' or donors' demographics, cold ischemic time, immunosuppression, pre-transplantation viral load, incidence of HBV infection, pre-transplantation diabetes mellitus, or incidence of biliary and vascular complications following transplantation.

\section{Impact of the IFNL3 polymorphism on antiviral treatment outcomes (SVR)}

Cure, defined as sustained virologic response (SVR24), was achieved in 29 of 76 (38.1\%) of the treated subjects.

In the case of the rs 127979860 genotype, recipients with the CC variant achieved SVR five times more often than recipients with the TT variant. If the donor had the same CC variant, the chance of achieving SVR was $100 \%$.

In the case of the rs12979860 genotype in the donor, no statistically significant differences were found. There were no statistically significant relationships between the rs8099917 polymorphism in the recipient and the donor, or between the combined recipient and donor polymorphism analysis and antiviral treatment outcomes. The above relationships are presented in Figures 1 and 2 .

\section{Impact of the IFNL3 polymorphism on the clinical course of HCV reinfection following liver transplantation}

An opposite impact of the same IFNL3 genotype on post-transplantation viral load was seen; it depended on whether it had been found in the recipient or the donor. Recipients with favorable (from the point of view of HCV eradication) CC variant of rs12979860 had the lowest levels of serum HCV RNA following

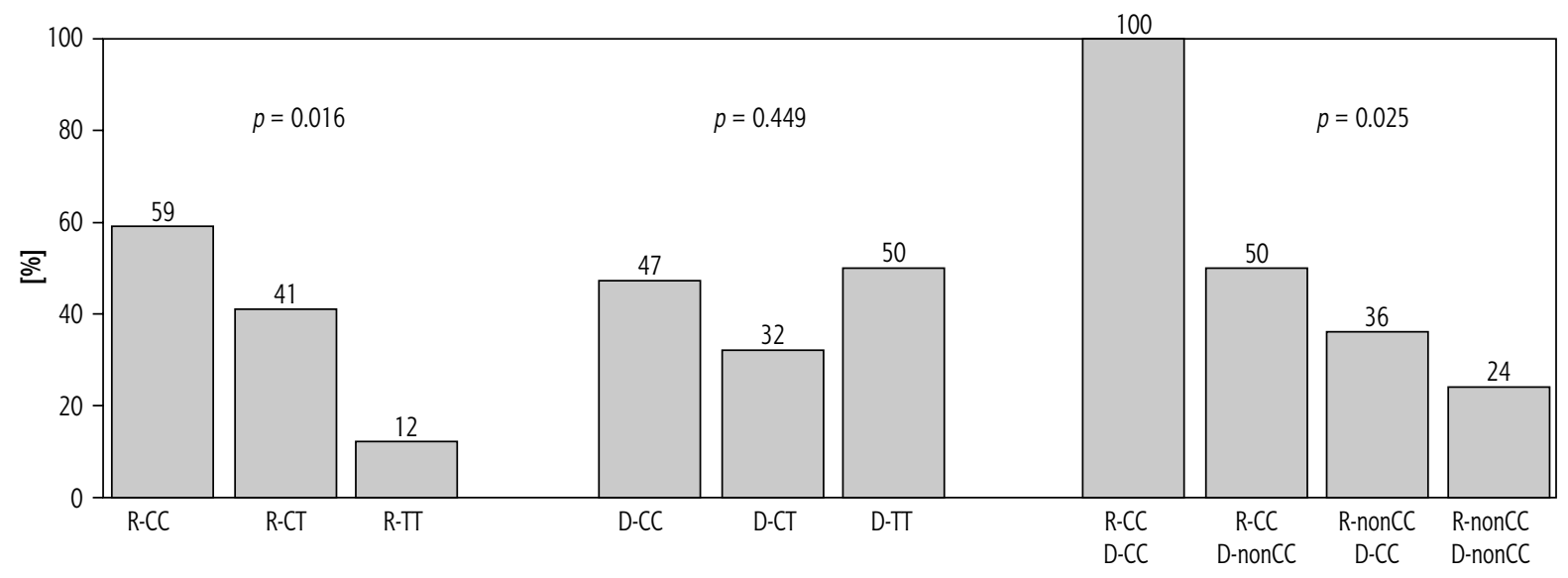

Fig. 1. Sustained virological response (SVR) rates by polymorphism rs12979860 (R-CC, R-CT, R-TT - variants of recipient's genotype, D-CC, D-CT, D-TT - variants of donor's genotype) 


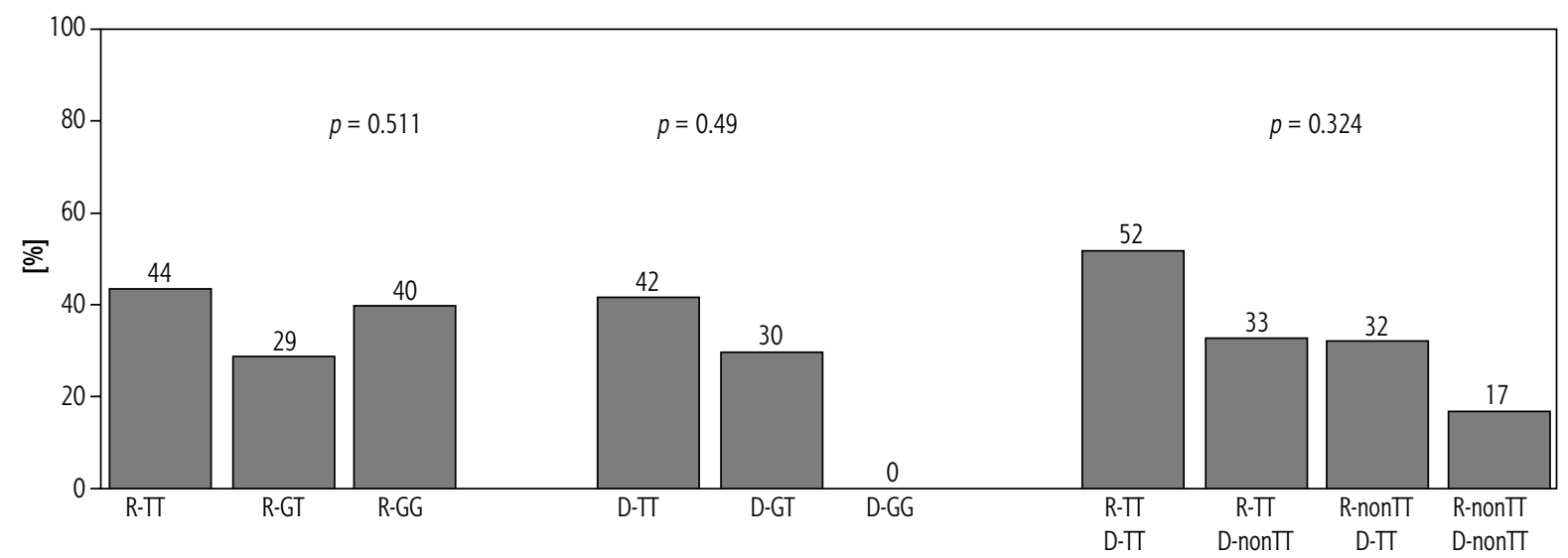

Fig. 2. Sustained virological response (SVR) rates by polymorphism rs8099917 (R-TT, R-GT, R-GG - variants of recipient's genotype, D-TT, D-GT, D-GG - variants of donor's genotype)

transplantation, as compared with other SNPs, and if the same variant had been found in the donor, viral load was the lowest. Similar relationships were reported for rs8099917, although statistically significant differences were reported only for the donor's genotype (Table 1). In a pooled analysis of both the recipient's and the donor's polymorphism, viral load was the highest for the combination of unfavorable (from the point of view of cure) HCV genotype of the recipient and favorable HCV genotype of the donor (for rs12979860, the combination: recipient - nonCC + donor-CC, $p=0.016$; for rs8099917, the combination: recipient - nonTT + donor - TT, $p=0.041$ ).

An analysis of mean serum activity of alanine aminotransferase (ALT) in post-transplantation year one, revealed an opposite impact of the recipient's and donor's genotype - as in the case of viral load: ALT levels were lower with the favorable genotype of the recipient and higher if the same genotype was found in the donor, although statistically significant differences were seen only for the donor's genotype for rs8099917 $(p=0.034)$, and - on the verge of statistical significance - for rs12979860 ( $p=0.051)$. The highest post-transplantation activity of ALT was seen with the combina- tion of an unfavorable recipient's genotype and favorable donor's genotype; at the same time, it is a group of patients with the highest post-transplantation viral load.

No statistically significant differences in the severity of inflammation and fibrosis stage by IFNL3 polymorphism were found, although similar tendencies as for viral load and ALT were seen: median inflammation and median fibrosis were higher in recipients with unfavorable IFNL3 polymorphism, especially if the donor had a favorable variant of the gene.

\section{Impact of the IFNL3 polymorphism on acute rejection rates following liver transplantation}

Acute rejection (AR) as evidenced by histological examination was diagnosed in 30 of 141 (21.3\%) study subjects. Lower AR rates were reported for carriers of unfavorable homozygotic variants of the gene (TT for rs12979860 and GG for rs8099917), although the differences were statistically significant only for the first SNP. The donor's genotype did not affect the rates of acute rejection. The relationship between acute rejection rates and IFNL3 polymorphism are presented in Figure 3.

Table 1. Hepatits C virus (HCV) viral load following transplantation by interferon lambda-3 (IFNL3) polymorphism in the recipient and the donor

\begin{tabular}{|c|c|c|c|c|c|c|}
\hline \multirow[t]{2}{*}{ SNP } & \multicolumn{6}{|c|}{ HCV RNA following transplantation } \\
\hline & Recipient & Median [1Q-3Q], $\log _{10^{\prime}} \mathrm{IU} / \mathrm{ml}$ & $p$-value & Donor & Median [1Q-3Q], $\log _{10^{\prime}} \mathrm{IU} / \mathrm{ml}$ & $p$-value \\
\hline \multirow[t]{3}{*}{ rs12979860 } & CC & $6.1[5.4-6.6]$ & \multirow[t]{3}{*}{0.011} & CC & $6.7[6.2-7.0]$ & \multirow[t]{3}{*}{0.001} \\
\hline & CT & $6.4[5.8-6.7]$ & & CT & $6.4[5.9-6.8]$ & \\
\hline & $\Pi$ & $6.6[6.2-7.1]$ & & $\Pi$ & $5.6[5.1-6.1]$ & \\
\hline \multirow[t]{3}{*}{ rs8099917 } & $\Pi$ & $6.2[5.7-6.6]$ & \multirow[t]{3}{*}{0.368} & $\Pi$ & $6.6[6.2-6.9]$ & \multirow[t]{3}{*}{0.002} \\
\hline & GT & $6.5[5.9-6.8]$ & & GT & $6.1[5.8-6.6]$ & \\
\hline & GG & 6.5 [6.1-6.9] & & GG & $5.3[5.2-5.3]$ & \\
\hline
\end{tabular}



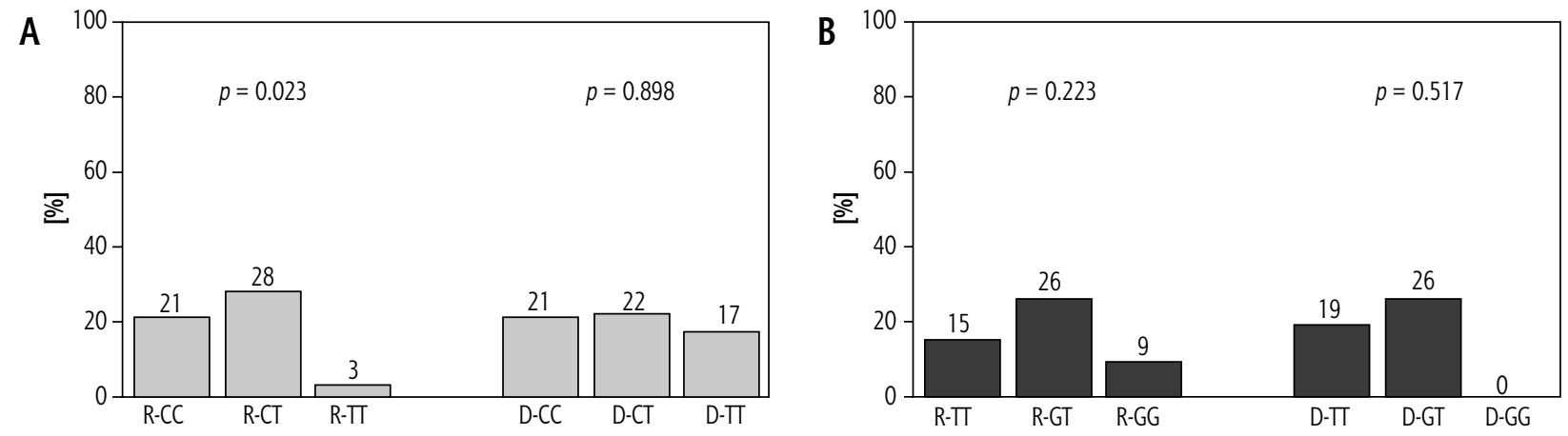

Fig. 3. Relationship between acute rejection rates and rs 12979860 (A) and rs8099917 (B) polymorphism (R - recipient, D - donor)
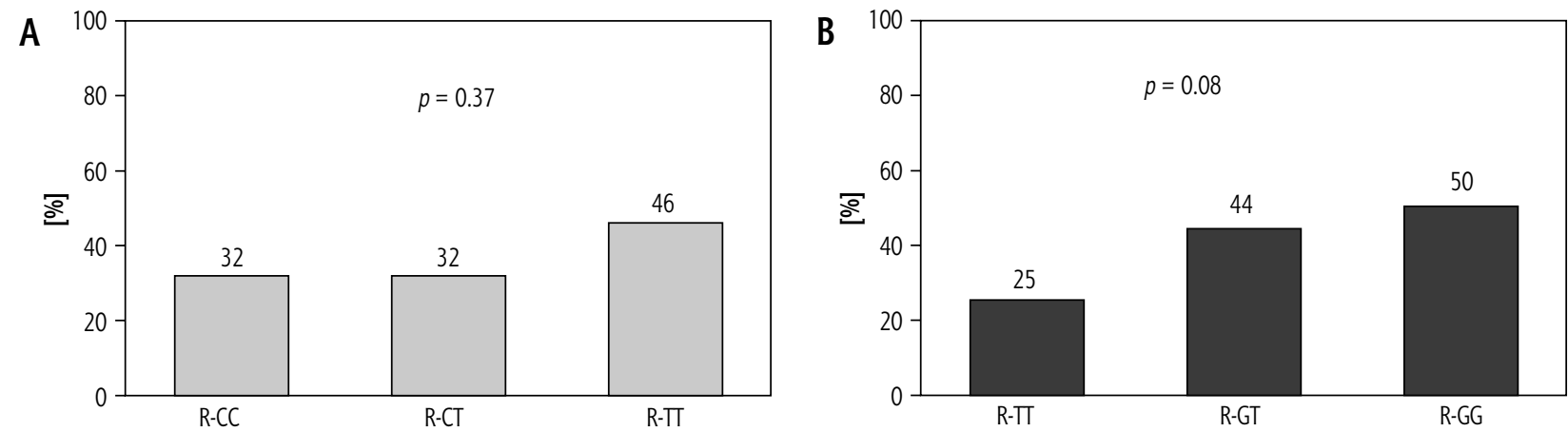

Fig. 4. The incidence of hepatocellular carcinoma (HCC) by the recipient's IFNL3 polymorphism: rs12979860 (A) and rs8099917 (B)

\section{The risk of hepatocellular carcinoma by IFNL3 genotype in the recipient}

Hepatocellular carcinoma in the patient's own liver was found in 46 of 141 (34.6\%) of the study subjects. It was demonstrated that the incidence of CC in the patient's own liver tended to be higher among subjects with unfavorable (from the point of view of $\mathrm{HCV}$ eradication) variants of the gene (Fig. 4).

\section{Discussion}

In the era of direct-acting antivirals (DAAs), IFNL3 polymorphism as the prognostic factor for assessing outcomes of $\mathrm{HCV}$ infection treatment is becoming less and less important, but it should not be forgotten altogether. IFNL3 genotyping enables the selection of patients with favorable IFNL3 polymorphism, which is predictive of high SVR rates for standard PegIFN- $\alpha$ / ribavirin-based therapy, comparable to SVR rates for new therapies. One may assume that in future, the problem will lie in the selection of mutated virus variants, which develop resistance to individual DAAs. Such mutations have already been reported in the con- text of clinical trials. Occurrence of new mutations is likely due to high genetic variability of HCV. Possibly, future treatment regimens may again include agents acting on the immune system of the host, such as interferon.

This paper demonstrates a surprising, opposite impact of the recipient's and donor's IFNL3 genotype on the clinical course of $\mathrm{HCV}$ reinfection following liver transplantation. A group of post-transplantation patients (unfavorable SNP of the recipient and favorable SNP of the donor) has been identified, in whom one may suspect a particularly aggressive HCV-reinfection of the liver graft associated with high viral load, high ALT activity, and an increased risk of progression of post-transplantation fibrosis. This group of patients requires urgent antiviral treatment following transplantation, preferably with DAAs. The cause of the opposite impact of the same SNP in both the recipient and the donor remains unclear. Similar relationships have been reported by other authors [16-18], however, no paper discusses these interesting though controversial results.

An explanation of these relationships may possibly be found in the paper by Chen et al. who detect- 
ed different expression of interferon-stimulated genes (ISG) in hepatocytes and hepatic macrophages in response to HCV infections. In the group of IFN/RBV responders, expression of basic interferon-stimulated genes (ISG15 and MxA) was relatively higher in macrophages and lower in hepatocytes. Unlike in non-responders, in whom high ISG induction in the liver and low in macrophages predominated [19]. Regrettably, the study by Chen et al. did not examine the IFNL3 polymorphism or correlation between the genotype, and ISG expression in individual cell types. However, the model described appears to perfectly apply to liver transplantation: the source of interferon lambda is composed of both the donor's hepatocytes and the recipient's immune cells, which populate the graft, and the acquired response of the recipient's macrophages is considerably impaired as a result of immunosuppression, while inborn response of the donor's hepatocytes is compromised only to a low degree. According to this theory, the highest viral load and ALT activity were found in the case of this combination: unfavorable recipient's genotype (low ISG expression in macrophages) and favorable donor's genotype (also low ISG in hepatocytes). On the whole, low levels of antiviral proteins would contribute to HCV replication and liver injury. The hypothetical model described would clearly require confirmation in studies examining correlation between the IFNL3 genotype with ISG expression in hepatocytes and macrophages. Nevertheless, it well explains the constellation of viral load and ALT activity in the study presented.

In this paper, also the relationship between IFNL3 polymorphism and acute rejection rates was examined. The rates of acute rejection following liver transplantation were lower among recipients with the most unfavorable (from the point of view of treatment efficacy) IFNL3 variants: rs12979860 TT and rs8099917 GG. This finding is surprising: few reports about a relationship between IFNL3 polymorphism and AR either failed to point the differences in rejection rates between SNPs or pointed to lower rejection rates in carriers of the favorable CC variant $[17,20]$. However, when the results are analyzed according to the model, which is based on the studies by Chen et al., one may assume that recipients of the unfavorable B-TT variant have low expression of pro-inflammatory cytokines in macrophages and other immune cells, which is why there may be lesser readiness to reject the donor's tissue, especially in the setting of immunosuppression. Taking such view explains also the lack of impact of the donor's genotype on the rejection process. Evidently, the concept presented should be considered with caution not only because of different findings of other authors, but also due to relatively low AR rates in the study group. On the other hand, its confirmation might have significant clinical implications: unfavorable polymorphism in the recipient at the same time promotes higher viral load and possibly more severe inflammation and greater damage to the liver graft. Therefore, more bold reduction of immunosuppression, without an increase of the rejection risk, might prove beneficial in this group of patients.

This paper, however, points to a tendency towards higher incidence of HCC in the liver of patients with unfavorable homozygotic variants of IFNL3: rs12979860 TT and rs8099917 GG, even though the differences were not statistically significant. A similar relationship between HCC and unfavorable SNPs has been reported by other authors $[21,22]$. At the cellular level, neogenesis to HCC is caused by chronic HCV-induced inflammation leading to overproduction of free oxygen radicals in hepatocytes, excited hepatic macrophages and the cells of inflammatory infiltrate. Chronic oxidative stress causes damage to cellular DNA and promotes neogenesis. It has been demonstrated that patients with unfavorable IFNL3 variants paradoxically have a higher expression of ISGs in hepatocytes, and therefore have more severe inflammation, which may increase the risk of neogenesis. It is worth considering whether variants TT for rs12979860 and GG for rs8099917 should be regarded as additional risk factors for HCC in patients with HCV-induced cirrhosis of the liver, and should increase oncological awareness when treating carriers of unfavorable SNPs. It is particularly important in light of the recent reports of higher incidence of hepatocellular carcinoma in patients after successful HCV eradication using new therapeutic regimes based on DAAs [23].

\section{Conclusions}

The IFNL3 rs12979860 polymorphism may be considered a predictor for IFN/RBV effectiveness following liver transplantation. Determination of the role of rs8099917 polymorphism in the Polish population requires further studies involving larger group of patients.

The course of HCV reinfection following liver transplantation may be more aggressive if an unfavorable variant in the recipient coexists with a favorable variant in the donor. Specific indications for urgent antiviral therapy after the transplantation should be considered in this group of patients.

Particularly careful oncological surveillance for HCC should be considered in patients with liver cirrhosis of HCV etiology, who are carriers of unfavorable IFNL3 variants. 


\section{Disclosure}

Authors report no conflict of interest.

\section{References}

1. Biggins SW, Bambha KM, Terrault NA, et al. Projected future increase in aging hepatitis $\mathrm{C}$ virus-infected liver transplant candidates: a potential effect of hepatocellular carcinoma. Liver Transpl 2012; 18: 1471-1478.

2. Forman LM, Lewis JD, Berlin JA, et al. The association between hepatitis $\mathrm{C}$ infection and survival after orthotopic liver transplantation. Gastroenterology 2002; 122: 889-896.

3. Neumann UP, Berg T, Bahra M, et al. Long-term outcome of liver transplants for chronic hepatitis C: a 10-year follow-up. Transplantation 2004; 77: 226-231.

4. Bunchorntavakul C, Reddy KR. Management of Hepatitis C Before and After Liver Transplantation in the Era of Rapidly Evolving Therapeutic Advances. J Clin Transl Hepatol 2014; 2: 124-133.

5. EASL Recommendations on Treatment of Hepatitis C 2015. J Hepatol 2015; 63: 199-236.

6. McHutchison JG, Lawitz EJ, Shiffman ML, et al. Peginterferon alfa- $2 b$ or alfa-2a with ribavirin for treatment of hepatitis $C$ infection. N Engl J Med 2009; 361: 580-593.

7. Reddy KR, Bunchorntavakul C. Managing hepatitis C: before and after liver transplantation. Indian J Transplant 2011; 5: 72-76.

8. Bunchorntavakul C, Bonnel AR, Reddy KR. Management of hepatitis before and after liver transplantation. In: Management of Hepatitis C: International Hepatology Updates Series. Arroyo V (ed.). Permanyer Publications, Barcelona 2011.

9. Suppiah V, Moldovan M, Ahlenstiel G, et al. IL28B is associated with response to chronic hepatitis $C$ interferon-alpha and ribavirin therapy. Nat Genet 2009; 41: 1100-1104.

10. Tanaka Y, Nishida N, Sugiyama M, et al. Genome-wide association of IL28B with response to pegylated interferon-alpha and ribavirin therapy for chronic hepatitis C. Nat Genet 2009; 41: 1105-1109.

11. Ge D, Fellay J, Thompson AJ, et al. Genetic variation in IL28B predicts hepatitis $\mathrm{C}$ treatment-induced viral clearance. Nature 2009; 461: 399-401.

12. Rauch A, Kutalik Z, Descombes P, et al. Genetic variation in IL28B is associated with chronic hepatitis $\mathrm{C}$ and treatment failure: a genome-wide association study. Gastroenterology 2010; 138: 1338-1345.

13. Balagopal A, Thomas DL, Thio CL. IL28B and the control of hepatitis $C$ virus infection. Gastroenterology 2010; 139: 1865-1876.

14. Ishak K, Baptista A, Bianchi L, et al. Histological grading and staging of chronic hepatitis. J Hepatol 1995; 22: 696-699.

15. Banff schema for grading liver allograft rejection: an international consensus document. Hepatology 1997; 25: 658-663.

16. Charlton MR, Thompson A, Veldt BJ, et al. Interleukin-28B polymorphisms are associated with histological recurrence and treatment response following liver transplantation in patients with hepatitis C virus infection. Hepatology 2011; 53: 317-324.

17. Duarte-Rojo A, Veldt BJ, Goldstein DD, et al. The course of posttransplant hepatitis $C$ infection: comparative impact of donor and recipient source of the favorable IL28B genotype and other variables. Transplantation 2012; 94: 197-203.

18. Lange CM, Moradpour D, Doehring A, et al. Impact of donor and recipient IL28B rs12979860 genotypes on hepatitis C virus liver graft reinfection. J Hepatol 2011; 55: 322-327.
19. Chen L, Borozan I, Sun J, et al. Cell-type specific gene expression signature in liver underlies response to interferon therapy in chronic hepatitis C infection. Gastroenterology 2010; 138: 1123-1133.

20. Duarte-Rojo A, Veldt BJ, Goldstein DD, et al. Recipient interleukin-28B Rs12979860 C/T polymorphism and acute cellular rejection after liver transplantation: role of the calcineurin inhibitor used. Transplantation 2012; 93: 1038-1044.

21. Suo GJ, Zhao ZX. Association of the interleukin-28B gene polymorphism with development of hepatitis virus-related hepatocellular carcinoma and liver cirrhosis: a meta-analysis. Genet Mol Res 2013; 12: 3708-3717.

22. Eurich D, Boas-Knoop S, Bahra M, et al. Role of IL28B polymorphism in the development of hepatitis $C$ virus-induced hepatocellular carcinoma, graft fibrosis, and posttransplant antiviral therapy. Transplantation 2012; 93: 644-649.

23. Reig M, Mariño Z, Perelló C, et al. Unexpected early tumor recurrence inpatients with hepatitis $\mathrm{C}$ virus -related hepatocellular carcinoma undergoing interferon-free therapy: a note of caution. J Hepatol 2016; 65: 719-726. 